\title{
A Multi-Agent Robot Coordination Framework for Goal based Navigation Problem
}

\author{
Mehmet Serdar Güzel \\ Computer Engineering Department \\ Ankara University, Turkey
}

\author{
İbrahim Baran Çelik \\ Computer Engineering Department \\ Ankara University, Turkey
}

\begin{abstract}
This paper addresses a vision based solution to collaborative multi-agent system for autonomous mobile robots. Each agent in the swarm is designed based on two different behavioural architectures, allowing them to overcome navigation problem in partly cluttered environment. A simple but efficient communication topology has been applied to the robot agents so as to run them in a swarm methodology. Robots' performance is being evaluated under different experimental conditions, and analysis of the results allows us to validate the proposed navigation approach.
\end{abstract}

Keywords—swarm robots, behavioral design, vision for robotics

\section{INTRODUCTION}

Swarm robotics has been an active and popular research area in terms of overcoming problems of collective movement which also gathers attention of robotic comity in all around the world $[1,2]$. The main motivation behind this project proposal is to provide the skills of being able to achieve different goal or goals with several robots simultaneously in a coordinated and planned manner. In mobile robotics applications, robot agents tend to run in an interacted manner to complete collective tasks such as task allocation and consensus achievement in an efficient way. Essentially collective work consumes less time for navigation, especially in goal-based navigation algorithms [3].

The Swarm robotics is mainly inspired from the nature, including how mammals or insects cooperate or interact with each other [4,5]. The group behaviour emerging in the swarm such as path planning and task allocation also resembles the behaviours of a robotics system. The swarm idea has also been applied to the human beings in whom a small informed minority can lead a group of unexperienced individuals towards a target in a shorter time with higher precision [6].

The multi-agent systems also inspired from the nature swarm which employs cooperative behaviour in order to achieve specific tasks [7]. In multi-agent system the size of the robot population is small and the robots and the environment is mostly known, in that respect, this study proposes a swarm solution to control a multi-agent system. Essentially, the aimed output of this study is to employ a swarm algorithm to complete the determined tasks in a short time and more efficient manner within coordination while preventing collision. In the first phase of the project, the robots registered into the system, relying on a real time image based target recognition algorithm, will explore the objects in a collective manner at the working environment.

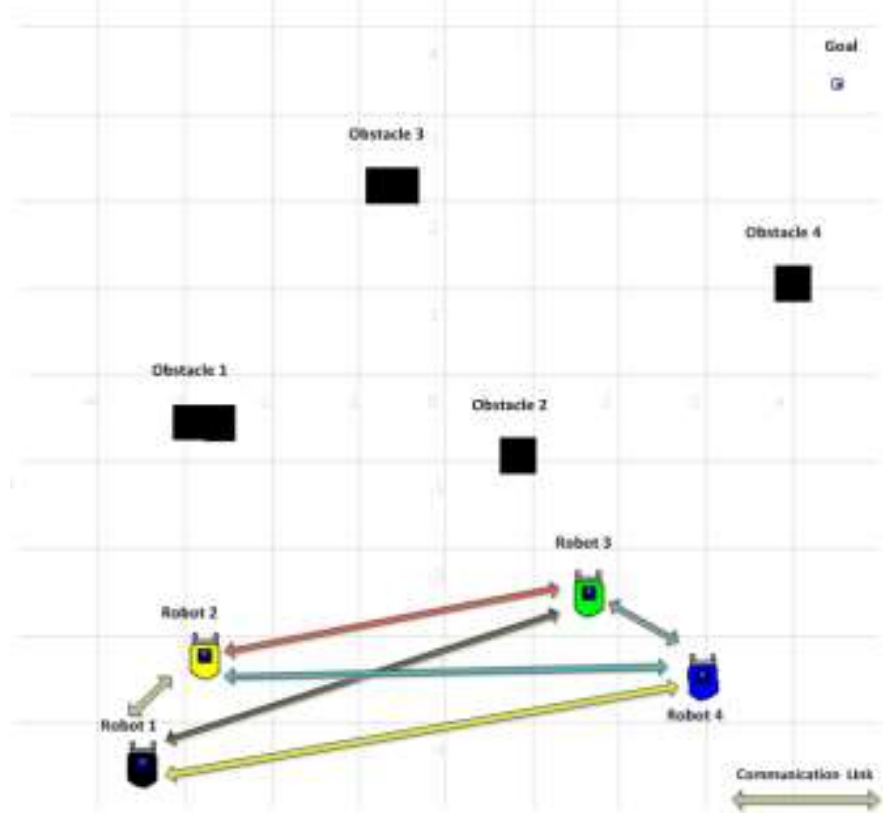

Fig. 1. An example scenario for multi-agent system

Once, any member of the robot swarm detects the position of the goal placed into the environment; it passes the location information to the other robots in order to provide them to complete the given task in an efficient manner.

Primarily, each robot in the swarm possesses two behavior based architecture. The first architecture is primarily responsible for detection of the goal which, in essence, involves three individual behaviors, namely 'goal_seek', 'message' and 'obstacle avoidance' behaviors. The second architecture, on the other hand allows robots to move towards a specific coordinates based on the hybrid based control architecture [8]. Within the scope of the proposed system, swarm robots will be able to search target or targets in coordinated and interacted manners.

The organization of the paper is defined in the following paragraph. Section 2 reviews address the proposed framework and corresponding algorithms, whereas section 3 focuses on 
the implementation and evaluation of the planned framework. Final section summarized the overall study.

\section{FRAMEWORK}

The proposed framework employs a decentralized control laws for autonomous mobile robots, which has been recently emerged as a challenging research area [5]. Primarily, in this control law, each robot interacts and communicates each other without requiring any central control unit. Besides, each robot in the proposed framework involves two behavior based architectures. In the first one, a behavior based architecture including three individual behaviors has been defined as previously mentioned in the introduction part. The second one, on the other hand, employs a hybrid based control architecture [8], both of which will be detailed in the following section.

The overall working scenario of the proposed system is illustrated in Figure 1 in which there are '4 robot agents', aiming to reach a specific target while avoiding obstacles placed in their ways. Once, one of these agents has reached the goal, it directly passes the coordinates of the goal to other agents so as to allow them to reach the goal. Essentially, all agents in the swarm need to communicate each other consistently about whether or not they reach the goal.

\section{A. Behavioural Design}

Each robot agent in the proposed framework is able to employ two behavioural based architecture as previously mentioned. Figure 2 illustrates the corresponding architectures.

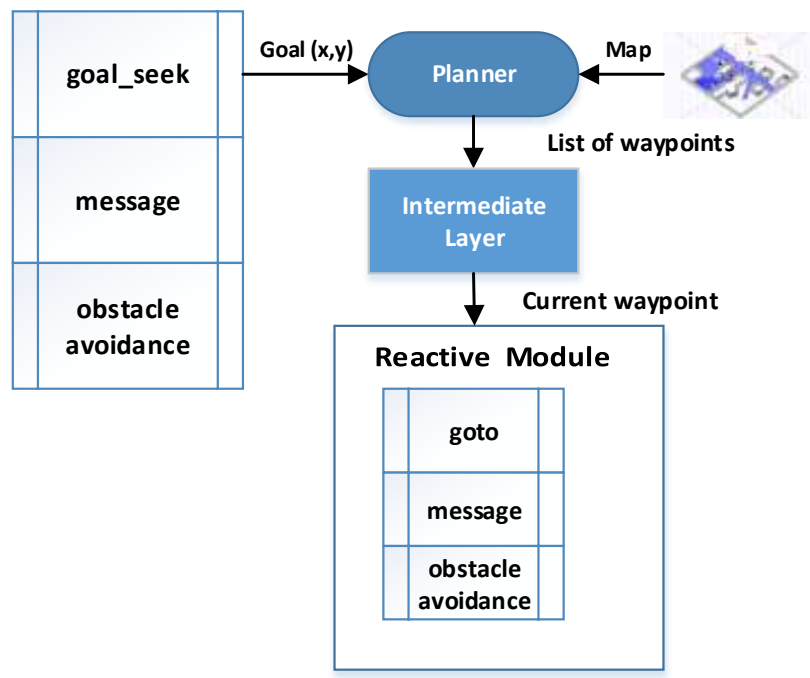

Fig. 2. Behavioural architecture of the robots

The first proposed architecture resembles the Brook's subsumption architecture [11] that each robot start to seek the goal using a vision based approach and notifies other agents via message behaviour. According to which, once the goal is found, the position of the goal is sent to other agents so as to lead them towards the goal.

The obstacle avoidance behaviour, on the other hand, has the highest priority (see Figure 2) and employs a range finder based Neural Network (NN) algorithm to overcome obstacles placed on the robot's way while seeking the goal. The detail of this $\mathrm{NN}$ based obstacle avoidance method can be seen in the corresponding study [9]. Once the goal is determined, the message, including the coordinate values of the goal, is passed to other agents and all corresponding agents in the swarm enable their proposed hybrid architecture to reach the goal.

The hybrid architecture involves a planner module which is responsible from global path planning. This module basically utilizes a 2-D world map of the given environment and the coordinates of the goal (see Figure 2). The planner module employs the Wavefront algorithm for planning phase that the algorithm generates a path from the start point ' $S$ ' to goal point ' $G$ '. The algorithm employs the breadth-first search algorithm on the graph, designed according to the neighborhood connectivity of cells [8]. Figure 3 illustrates how the Wavefront algorithm works.

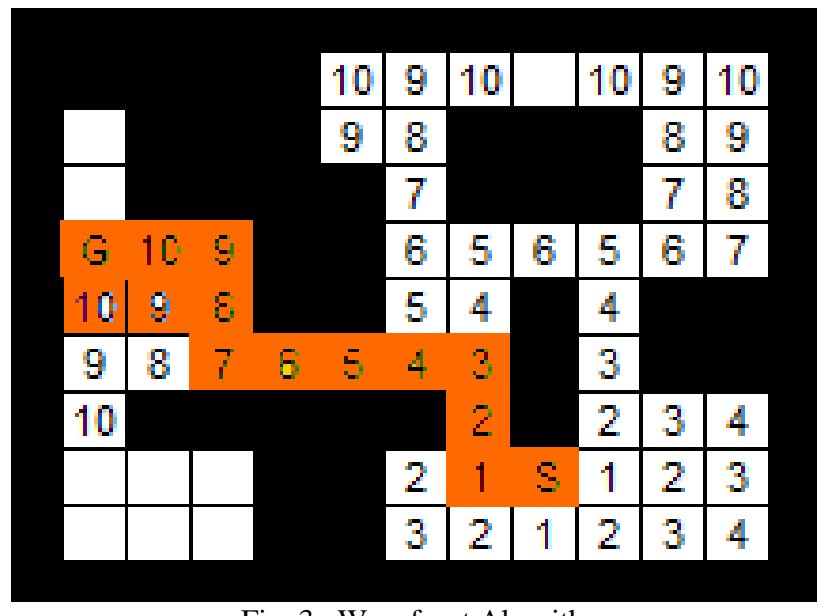

Fig. 3. Wavefront Algorithm

The waypoints are then accepted by the intermediate layer, and pass to the reactive module successively for execution. The reactive module has been designed for local navigation problem. This module primarily employs VFH (Vector Field Histogram) [10] algorithm to reach the current waypoint while avoiding obstacles placed on its path. The VFF algorithm essentially employs 2-D histogram data as the world model based on range finder sensors. Besides, the algorithm mainly comprises two steps in order to calculate steering parameters for the autonomous vehicle toward a specific target. In the first step, the histogram grid is reduced into one dimensional polar histogram. At the following step, the sector with the lowest density value is selected from the polar histogram and the steering of the mobile agent is obtained according to this direction [10].

Despite its high performance, VFH has some shortcomings and may cause collision with obstacles, 
especially in cluttered environment. Hereby, an alternative obstacle avoidance behavior [9] is added into the reactive module. This behavior has the highest priority and is enabled as the VFH cannot handle the obstacles nearby. The middle layer of the reactive module, on the other hand, is the message layer which provides 'instant communication' between the current and the other agents.

\section{IMPLEMENTATION OF THE SYSTEM}

The system has been implemented using Player/Stage robotic software tool [12]. The Stage is a 2-D simulator tool without any camera support. Accordingly, OpenCV, computer vision toolbox, has been integrated into this simulator in order to overcome camera based navigation problem. The primary system environment of the simulator for the given problem is illustrated in Figure 4.

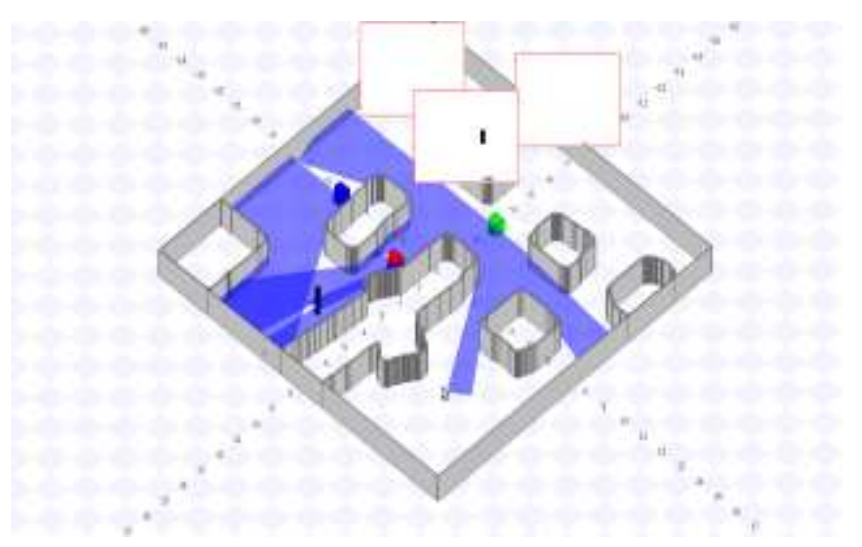

Fig. 4. Simulation Environment

Robots are equipped with a camera, and a colour based blob detection algorithm has been applied so as to detect the targets with minimum effort. As well as, a 2-D laser range finder has been integrated into the agents for obstacle avoidance problem, which is far more precise than the sonar range finder.

\section{A. Experimental Design and Evaluation}

The associated system has been implemented on the Stage simulator. The overall performance of this system has been evaluated under different experimental conditions. Three different scenarios have been illustrated in this section.

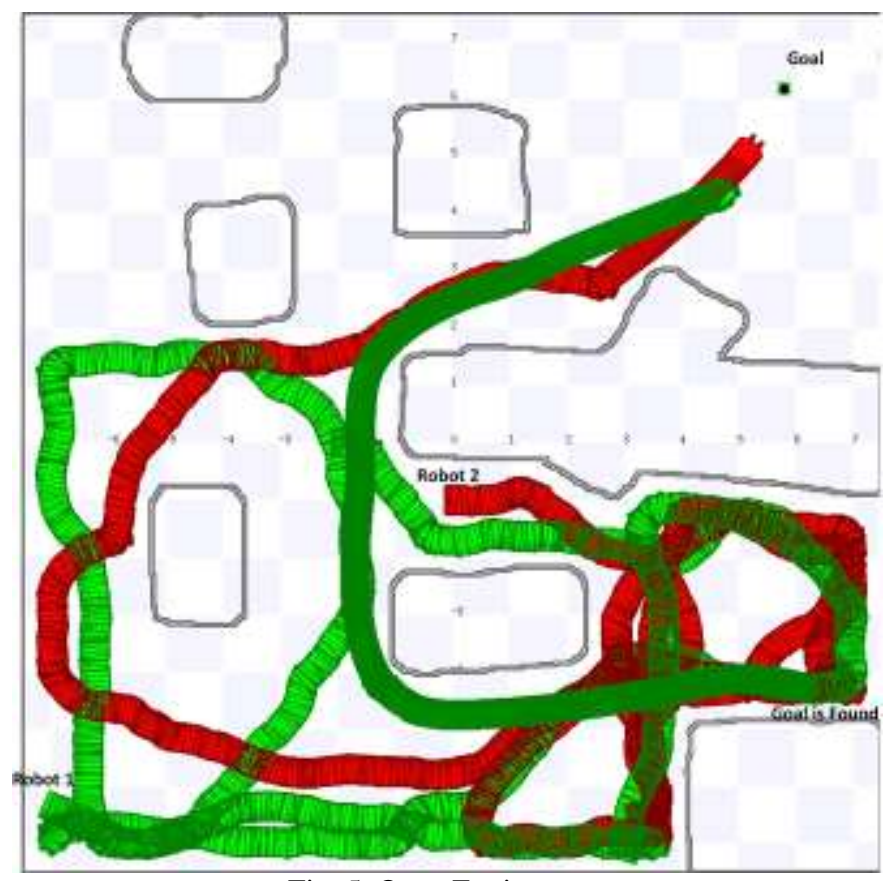

Fig. 5. Open Environment

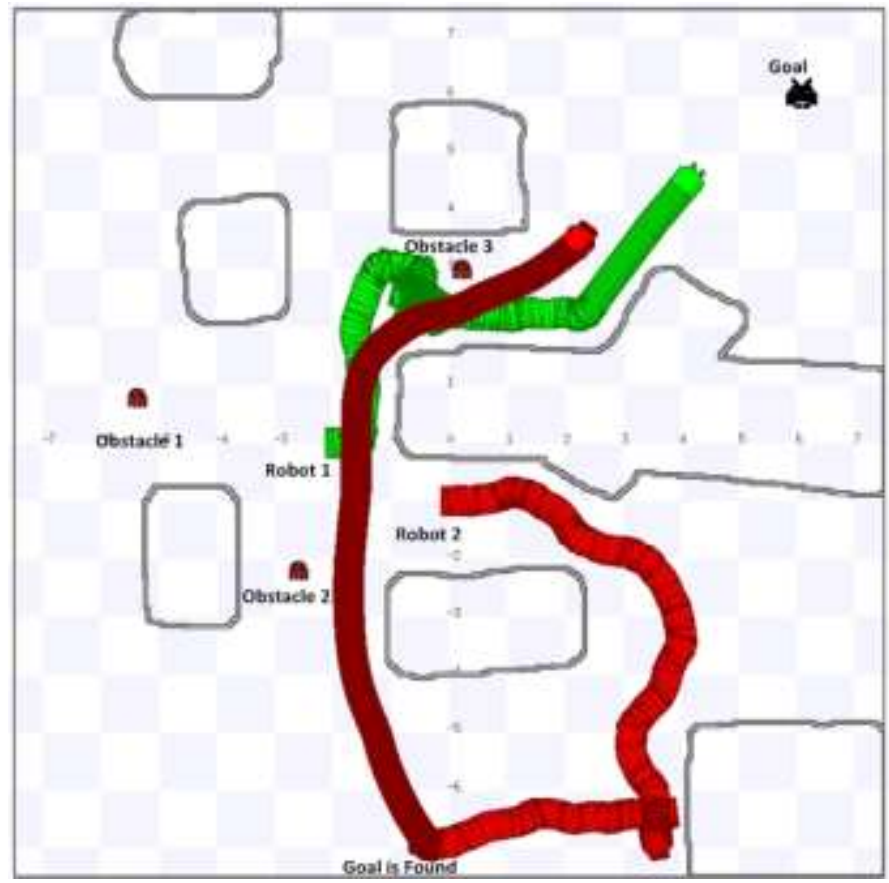

Fig. 6. Test Environment with static obstacles

Figure 5 demonstrates a scenario in which the robots try to navigate in a partially cluttered environment towards an unknown goal (open environment). According to the scenario, the red and green robots start to explore the goal while trying to avoiding collision. Once the goal is detected (found) by the red robot's vison based detection system, the coordinate value of the goal is transferred to the green one, which then navigates towards the goal deliberately (illustrated with dark green track at the Figure 5). The second scenario involves an environment including static obstacles and two robots explore 
the goal while trying to avoid collision with the environment and the static obstacles placed on the path of the robot as shown in Figure 6.

For the second scenario, the robots placed different start points initially start searching for the goal. The green robot is able to successfully find the goal without colliding the wall and the Obstacle (Obstacle 3) as illustrated in Figure 6. When the goal is found by the green robot, the position of the goal is transferred to the red robot as expected. Afterwards, this robot employs the hybrid architecture (see 2.A) to reach the goal. The trajectory of the red robot towards the goal is illustrated with dark red track that the robot first employs the Wavefront algorithm to calculate waypoints, followed by employing the reactive module to reach each corresponding waypoint.

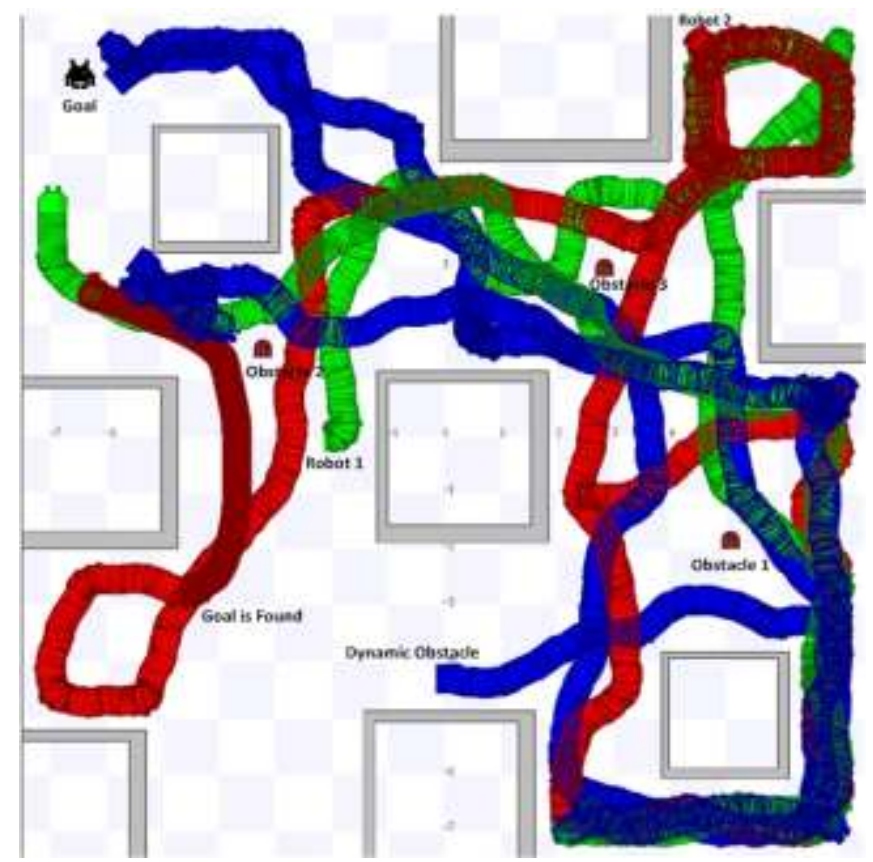

Fig. 7. Test Environment with static and dynamic obstacles

Third scenario represents a more cluttered environment comprising two static obstacles and one dynamic obstacle (blue robot). The robots are required to navigate from the start point to the goal with obstacles positioned so as to obstruct their path. The blue robot is assigned as the dynamic obstacle which moves randomly along the environment. The robots are trying to avoid collision with both the static and the dynamic obstacles as illustrated in Figure 7. Once, the green robot detects the position of the goal, the red one is informed and led toward the goal as illustrated with the dark red track at the corresponding figure.

Final scenario, illustrated in Figure 8, represents the performance of three robot agents trying to reach a predefined goal whilst avoiding static obstacles. According to the scenario, once the green robot (robot 3 ) detects the goal, it primarily starts moving towards the goal deliberately, followed by conveying the position information of the goal to the other agents in order to lead them towards the goal.
The proposed navigation architecture can be applied from 2 to $\mathrm{n}$ robots in a multi agent system for different problems. The results reveal that this architecture proposes a simple but efficient way for multi agent systems that decreases time consumed to complete the given task. As it is expected, the overall performance gain of the system is proportionally increased with the increase in robot agent numbers, registered to swarm.

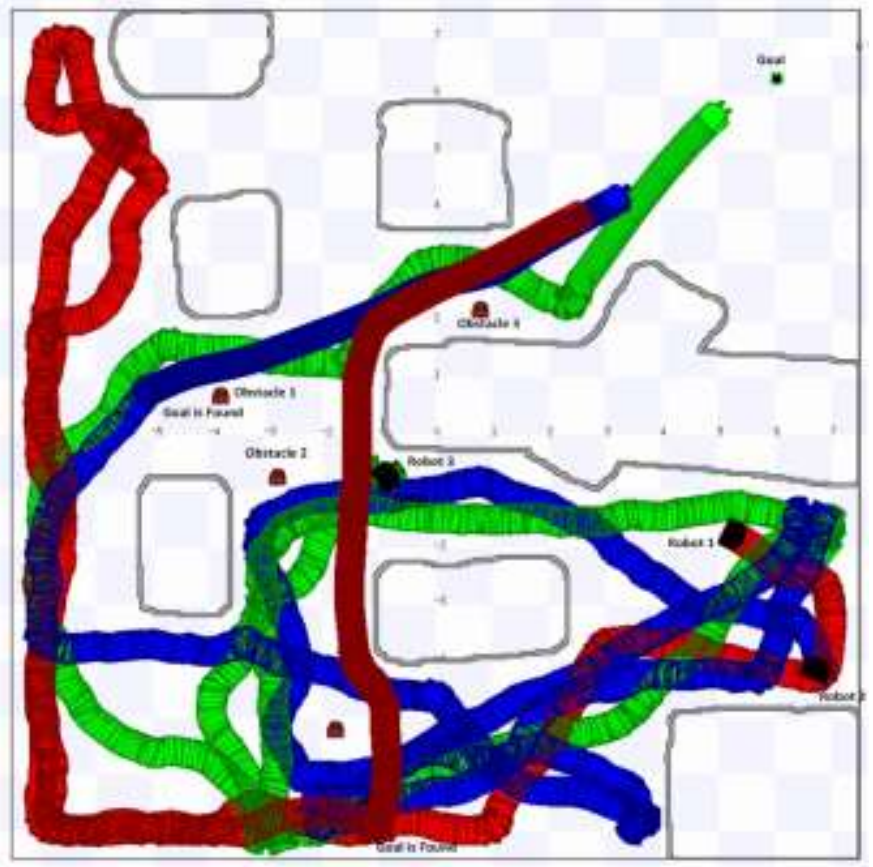

Fig. 8. Test Environment with static for "3" robots

\section{Conclusions}

This paper describes a new behaviour-based architecture for multi-agent systems. The architecture involves two different behavioural architecture. The first architecture involves a vision based goal seeking strategy that each robot registered to the swarm employs a reactive architecture to detect the goal. Once the goal is detected by any of the agent, other agents are immediately informed by the corresponding robot about the position of the goal. First the corresponding robot and then other agents in the swarm start to employ the hybrid architecture to reach the goal in a more deliberate strategy.

The system has been tested under different experimental conditions using a popular simulator and results are quite encouraging. As well as, several improvements in the architecture could produce more reliable results and the system can then be integrated into many areas from defence industry to rescue robots.

\section{References}

[1] Beni, G.; Wang, J., S., "Intelligence in Cellular Robotic Systems", Proceed. NATO Advanced Workshop on Robots and Biological Systems, Tuscany, Italy, June 26-30, (1989). 
[2] Kazadi, S.; "Swarm Engineering", PhD thesis, California Institute of Technology, Pasadeba, CA, USA, (2000).

[3] Spears, W., M.; Spears, D., F.; Hamann, J., C.; and Heil, R., "Distributed, physics-based control of swarms of vehicles. Autonomous Robots", 17(2-3):137-162 (2004).

[4] Bonabeau E, Dorigo M, Theraulaz G., "From natural to artificial swarm intelligence", Oxford: Oxford University Press; (1999).

[5] Camazine S, Deneubourg JL, Frank NR, Sneyd J, Theraulaz G, Bonabeau E. "Self-organization in biological systems", Princeton: Princeton University Press; (2003).

[6] Dyer JRG, Ioannou CC, Morrell LJ, Croft DP, Couzin ID, Waters Dean A, et al., "Consensus decision making in human crowds", Anim Behav;75(2 ), (2008)

[7] Yan Ying and Zheng Zhong-yang, "Research Advance in Swarm Robotics", Defence Techonlogy 9, pp:18-39, (2013).

[8] Nattharith, P., "Behaviour Modulation using Fuzzy Logic Control for Mobile Robot Navigation", International Journal of Engineering and Physical Sciences, Vol. 6, p. $341-347$, (2012).

[9] M. S. Guzel and R. Bicker, "A Behaviour-based Architecture for Mapless Navigation using Vision", International Journal of Advanced Robotic Systems, vol. 9, pp. 1-13,(2012).

[10] J. Borenstein and Y. Koren, "The Vector Field Histogram - Fast Obstacle Avoidance for Mobile Robots", IEEE Transactions on Robotics and Automation, 7(3), (1991).

[11] Brooks, R. A. (1986) 'Robust Layered Control System for a Mobile Robot', IEEE Journal of Robotics and Automation, RA-2, (1), pp. 14-23.

[12] B. P. Gerkey, R. T. Vaughan, and A. Howard, "The player/stage project: Tools for multi-robot and distributed sensor systems," in Proceedings of the International Conference on Advanced Robotics, Coimbra, Portugal, Jul, pp. 317-323, (2003).

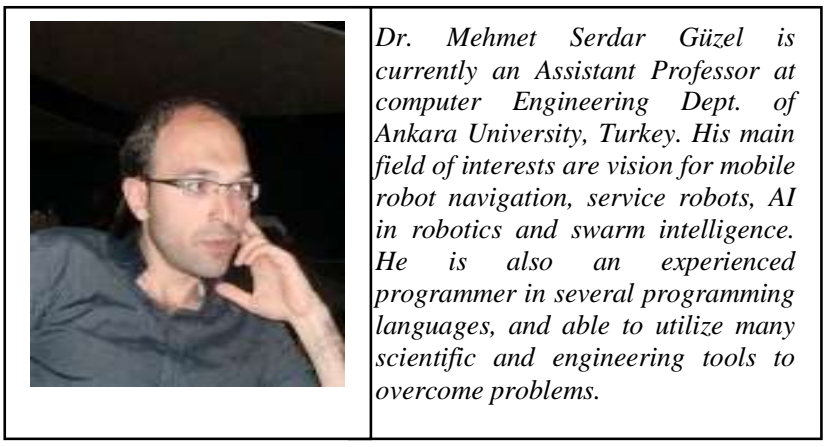

\begin{tabular}{|l|l|}
\hline 5 & $\begin{array}{l}\text { Mr. Çelik is currently a PhD Student at the } \\
\text { computer Engineering Dept. Of Ankara } \\
\text { University, Turkey. He has also been working } \\
\text { as a software engineer at private companies, } \\
\text { mainly active at defence industry. His main } \\
\text { field of interests are robotics, embedded } \\
\text { systems and software development }\end{array}$ \\
\hline
\end{tabular}

Mr. Çelik is currently a PhD Student at the University, Turkey. He has also been working as a software engineer at private companies, mainly active at defence industry. His main systems and software development 